\title{
Radio frequency-vacuum drying of kiwifruits: kinetics, uniformity and product quality
}

\author{
Wang, S. ${ }^{\text {ab*; Zhou, }}$ X. $^{\text {a }}$ \\ ${ }^{a}$ College of Mechanical and Electronic Engineering, Northwest A\&F University, Yangling, Shaanxi \\ 712100, China \\ b Department of Biological Systems Engineering, Washington State University, 213 L.J. Smith Hall, \\ Pullman, WA 99164-6120, USA
}

*E-mail of the corresponding author: shaojinwang@nwsuaf.edu.cn

\begin{abstract}
A radio frequency $(R F)$ vacuum technology is proposed for drying kiwifruit slices using a $27.12 \mathrm{MHz}, 3 \mathrm{~kW}$ RF-vacuum drying system. The results demonstrated that electrode gap, vacuum pressure and sample thickness had major effects on the RF-vacuum drying. The RF-vacuum drying was associated with internal heating and rapid drying, resulting in $65 \%$ reduction of hot air $\left(60^{\circ} \mathrm{C}\right)$ drying time. Moreover, kiwifruits dehydrated by RF-vacuum drying were associated with better color stability, higher vitamin $C$ retention and higher rehydration capacity $(P<0.05)$. Overall, the $R F$-vacuum drying process may provide a more effective and practical method for high-quality dehydration of kiwifruits.
\end{abstract}

Keywords: radio frequency-vacuum drying; hot air; moisture content distribution; moisture effective diffusivity; quality 


\section{Introduction}

Kiwifruit (Actinidia deliciosa) is recognized as "the king of fruits" due to its remarkably high amounts of vitamin $\mathrm{C}$ and bioactive compounds with antioxidant activity. The global production of kiwifruit was around 4.27 million metric tons (Mt) in 2016 mainly contributed by China (2.39 Mt), Italy (0.52 Mt), New Zealand (0.43 Mt) and Chile (0.23 Mt). ${ }^{[1]}$ However, kiwifruit is a seasonal fruit being harvested during August-October in China. Owing to high moisture content, fresh kiwifruit is highly perishable after harvest, even under refrigerated storage conditions. Thus, developing a proper postharvest drying technique is an important consideration to overcome the problems of seasonality and extend kiwifruit shelf-life to protect farmers' income and local economy.

The most conventional drying methods for kiwifruits, such as osmotic dehydration and hot air drying, have low technical barriers in developing/undeveloped countries and are preferred in industry, but with intensive labor cost, high energy consumption and long drying time. ${ }^{[2]}$ Microwave (MW) and radio frequency (RF) drying methods also known as dielectric heating generate heat within the food material by molecular friction as a result of dipolar rotation and ionic conduction. ${ }^{[3,4]} \mathrm{RF}$ heating has received increasing attention over the past decade due to its longer wavelength and deeper wave penetration as compared with MW and is thereby technologically more feasible for industrial applications. ${ }^{[5]}$ Furthermore, the use of vacuum in RF heating, resulting in RF-vacuum drying, may further raise drying rate and ensure high quality in the final product because of the reduced drying temperatures as well as oxygen potential and considerably higher vapor pressure gradients between the interior and the surface of materials.

Therefore, the objectives of this study were (1) to determine the effect of RF process variables and sample thickness on the RF-vacuum drying characteristics of kiwifruit slices, (2) to study the drying kinetics and effective moisture diffusivity of hot air and RF-vacuum drying, and (3) to evaluate the quality of dried kiwifruit samples in terms of moisture content uniformity, color, vitamin C content and rehydration capacity.

\section{Materials and Methods}

\subsection{Materials and RF-vacuum drying system}

Freshly harvested kiwifruits (Actinidia deliciosa cultivar "Hayward") observed visually for similar ripeness and size were obtained from Shaanxi, China. The fruits were then hand peeled and cut into slices with diameter $45.5 \pm 5.4 \mathrm{~mm}$ and three thicknesses: $6.1 \pm 0.2,8.0$ \pm 0.2 and $10.0 \pm 0.3 \mathrm{~mm}$. 
A $3 \mathrm{~kW}, 27.12 \mathrm{MHz}$ free-running oscillator RF-vacuum drying system (GJ-3-27-JY, Jiyuan High Frequency Electric, Shijiazhuang, China) was used for RF-vacuum drying experiments (Fig. 1). The RF electrode gaps between the two parallel plates (400 $\mathrm{mm} \times 400 \mathrm{~mm}$ ) could be adjusted from $20 \mathrm{~mm}$ to $300 \mathrm{~mm}$ to deliver desired RF energy for specific applications. The system pressure (vacuum) in the RF chamber, and sample temperature and mass were continuously recorded by a pressure sensor (APC500, Sensor Way Technologies Inc., Beijing, China) located in the vacuum cavity, four-channel fiber-optic sensor system (HQFTS-D120, Heqi Technologies Inc., Xian, China), an electronic scale (AT8106, Pengheng Electronic Inc., Shanghai, China) with a precision of $0.1 \mathrm{~g}$ mounted underneath the bottom electrode, respectively, during the entire drying process.

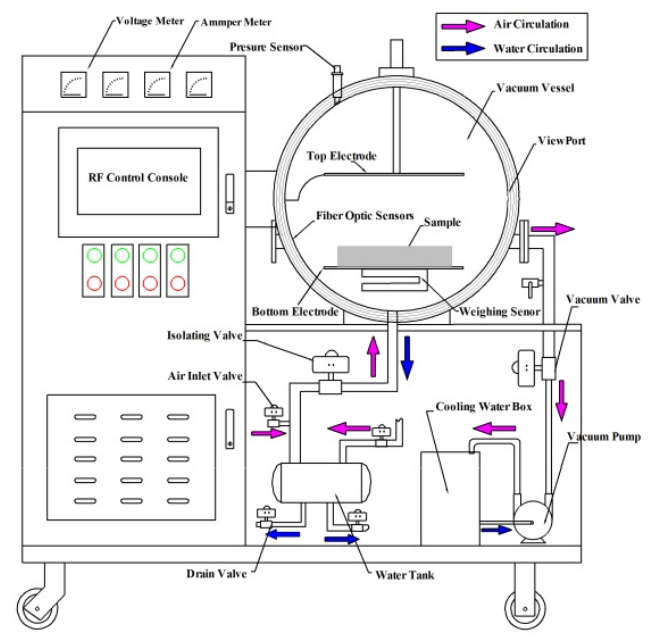

Fig. 1 Schematic view of the $3 \mathrm{~kW}, 27.12 \mathrm{MHz} R$ F-vacuum drying system

\subsection{Moisture content determination and drying procedures}

The moisture content of kiwifruit slices was determined following the AOAC Official Method 925.40 and expressed as g[water]/g[solid] through the drying process.

RF-vacuum drying: Twenty-four freshly prepared kiwifruit slices (523.4 \pm 18.0 g) were placed uniformly in a single layer inside a container (400 mm $L \times 270 \mathrm{~mm} W \times 20 \mathrm{~mm} \mathrm{H}$ ) made of polypropylene (PP) with its side and bottom walls perforated with $10 \mathrm{~mm}$ diameter holes. Three electrode gaps (50, 60 and $70 \mathrm{~mm}$ ) with three vacuum pressure levels $(0.01,0.02$ and $0.03 \mathrm{MPa})$ and three sample thicknesses $(6,8$ and $10 \mathrm{~mm})$ were selected as process/product variables for determining RF-vacuum drying characteristics. The sample temperature and mass were continuously recorded by the fiber-optic temperature sensors and the electronic scale without having to turn off RF power or taking out samples. The RF- 
vacuum drying process was continued until the moisture content of kiwifruit samples reached $0.18 \mathrm{~kg} / \mathrm{kg}$ (d.b.)

\subsection{Mathematical modeling of drying curves}

The transient changes in moisture content of kiwifruit samples during drying was expressed as moisture ratio $(M R)$ defined as:

$$
M R=\frac{M_{\mathrm{i}}-M_{\mathrm{e}}}{M_{\mathrm{o}}-M_{\mathrm{e}}}
$$

where $M R$ is the dimensionless moisture ratio, $M_{\mathrm{i}}$ or $M_{\mathrm{i}-1}(\mathrm{~kg} / \mathrm{kg}$, d.b.) is the moisture content at any time $i$ or $i-1, \Delta t$ is the drying time interval between time $i$ and $i-1(\mathrm{~min}), M_{0}$ is the initial moisture content $(\mathrm{kg} / \mathrm{kg}$, d.b.) and $M e$ is the equilibrium moisture content $(\mathrm{kg} / \mathrm{kg}$, d.b.).

\subsection{Evaluation of product quality}

\subsubsection{Moisture content uniformity}

Moisture content uniformity was moisture distribution among dried kiwifruit slices in the container. The moisture contents of kiwifruit slices located at 10 representative container locations were measured after drying.

\subsubsection{Color}

The color $\left(L^{*}, a^{*}, b^{*}\right)$ of fresh (control) and dried kiwifruit slice samples was measured using a computer vision system. The total value of color difference $(\Delta E)$ was calculated as follows:

$$
\Delta E=\sqrt{\left(L^{*}-L_{0}^{*}\right)^{2}+\left(a^{*}-a_{0}^{*}\right)^{2}+\left(b^{*}-b_{0}^{*}\right)^{2}}
$$

\subsubsection{Vitamin C (ascorbic acid) content}

Ascorbic acid content was determined by the standard 2,6-dichloroindophenol titration method.

\subsubsection{Rehydration capacity}

Rehydration capacity of dried kiwifruit slices was measured according to the method of Maskan (2001) ${ }^{[6]}$

$$
\text { Rehydration capacity }=\frac{W-W_{0}}{W_{0}} \times 100 \%
$$

where $W_{0}$ and $W$ are the sample weight values (g) before and after rehydration, respectively. 


\section{Results and Discussion}

\subsection{RF-vacuum drying and heating characteristics}

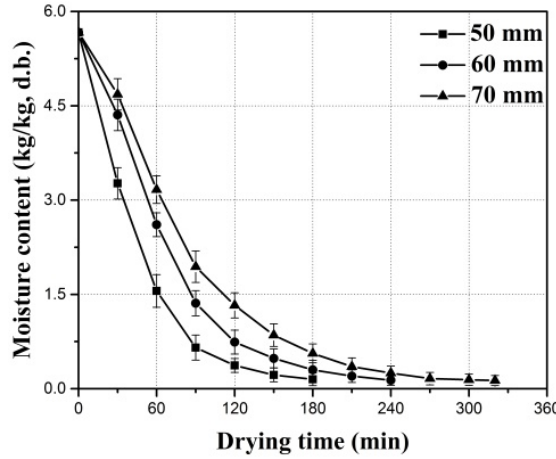

(a)

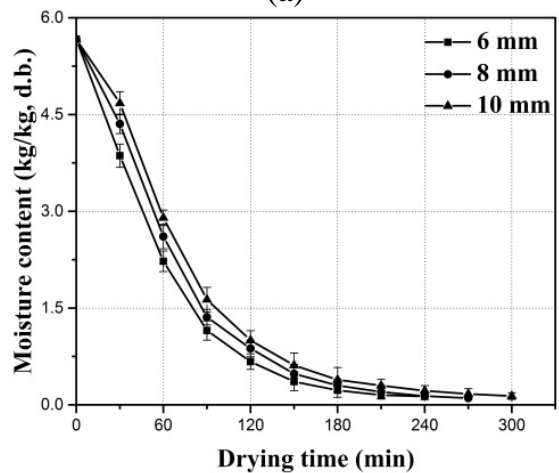

(c)

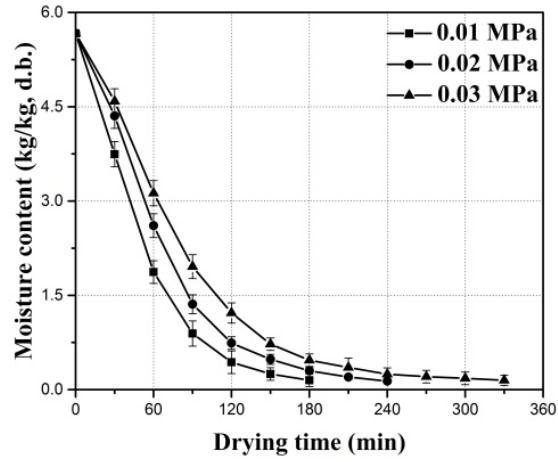

(b)

Fig. 2 RF-vacuum drying characteristics of kiwifruits influenced by electrode gap (a), vacuum pressure (b) and sample thickness (c)

The electrode gap, vacuum pressure and sample thickness had significant effects on RFvacuum heating and drying characteristics of kiwifruit slices (Fig. 2). The RF-vacuum drying profile involved three stages regardless of various operating parameters: Stage I in which RF energy was transformed into thermal one within the materials and sample temperature rapidly increased. Once the moisture vapor pressure in the samples exceeded that of environment, the moisture expulsion process got and the drying rate gradually increased. Stage II began when the product temperature reached the highest value (wet bulb temperature), the drying process entered into a rapid drying period (constant drying rate period) and the sample temperature remained at a fairly constant level. As the drying progressed into Stage III, the loss of water in the samples reduced the associated dielectric properties, resulting in reduced 
absorption of RF energy, and led to a gradually decreasing rate of drying toward the final stage of drying. Additionally, the thermal energy required for breaking away bound water is higher than that required for free water. Consequently, the RF-vacuum drying rate gradually decreased and the drying process was dominated by the falling drying rate period (Stage III). In general, the RF-vacuum drying process may limit the temperature raise in the samples and reduce severe thermal deterioration of product quality.

Fig. 3 shows a typical drying temperature-time profile at the core and sub-surface of kiwifruit slices subjected to RF-vacuum and hot air drying, indicating that the RF-vacuum dried materials were heated throughout the sample whereas surface higher temperatures were found in hot air drying.

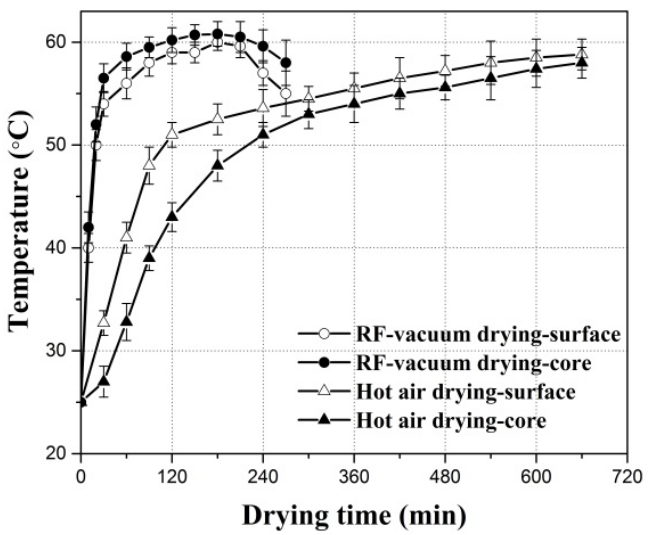

Fig. 3 Typical temperature-time history for sub-surface $(2 \mathrm{~mm})$ and core $(20 \mathrm{~mm})$ of kiwifruit slices when subjected to hot air and RF-vacuum drying

\subsection{Drying kinetics and moisture effective diffusivity}

The curves for $M R$ versus drying time with the best drying models under hot air and RFvacuum drying are shown in Fig. 4. The Page model ( $R^{2}$ of 0.9998 and $R M S E$ of 0.0056$)$ was the best fit to describe hot air drying while the logarithmic model ( $R^{2}$ of 0.9977 and $R M S E$ of 0.0145 ) provided the best fit for RF-vacuum drying. Generally, the drying time for kiwifruit slices was reduced by about 65\% when using RF-vacuum drying technology as compared to hot air drying, highlighting the advantage associated with RF-vacuum drying technology. 
Wang, S.; Zhou, $X$.

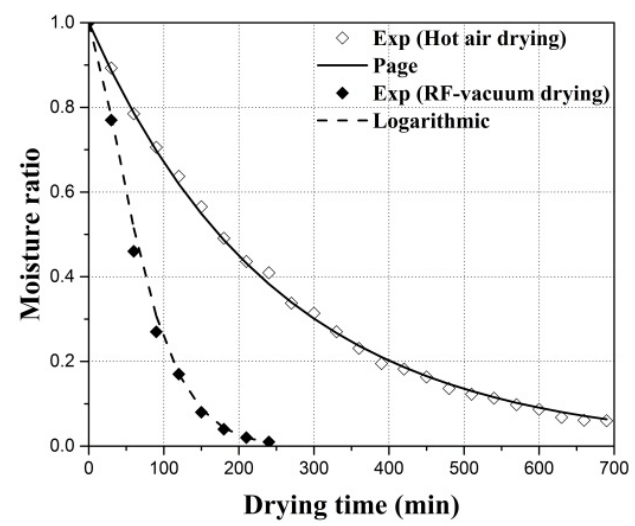

Fig. 4 Drying kinetics of kiwifruit slices when subjected to hot air and RF-vacuum drying

\subsection{Quality of dried kiwifruit slices}

Table 1 quality characteristics of kiwifruits before and after drying

\begin{tabular}{lllllll}
\hline & $L^{*}$ & $a^{*}$ & $b^{*}$ & $\Delta E$ & $\begin{array}{l}\text { Vc content } \\
(\mathrm{mg} / 100 \mathrm{~g})\end{array}$ & $\begin{array}{l}\text { RC } \\
(\%)\end{array}$ \\
\hline Fresh & $42.2 \pm 1.0 \mathrm{a} \#$ & $-8.9 \pm 0.1 \mathrm{c}$ & $24.7 \pm 0.4 \mathrm{a}$ & - & $115.3 \pm 6.5 \mathrm{a}$ & - \\
AD & $29.7 \pm 3.9 \mathrm{c}$ & $-0.8 \pm 0.8 \mathrm{a}$ & $20.4 \pm 0.6 \mathrm{c}$ & $15.5 \pm 1.4 \mathrm{a}$ & $45.8 \pm 5.6 \mathrm{c}$ & $115.8 \pm 4.5 \mathrm{~b}$ \\
RF & $36.4 \pm 2.2 \mathrm{~b}$ & $-5.4 \pm 0.4 \mathrm{~b}$ & $22.2 \pm 0.4 \mathrm{~b}$ & $7.1 \pm 0.8 \mathrm{~b}$ & $62.5 \pm 4.7 \mathrm{~b}$ & $148.4 \pm 6.8 \mathrm{a}$ \\
\hline
\end{tabular}

Table 1 summarizes the results for kiwifruit product quality after hot air and RF-vacuum drying. The total color change $(\Delta E)$ of RF-vacuum dried kiwifruits was significantly smaller $(\mathrm{P}<0.05)$ possibly due to the shorter drying time, lower temperatures and reduced oxygen concentration. The retention ratio of vitamin $C$ was less than $60 \%$ or lower ( $54 \%$ and $40 \%$ for RF-vacuum and hot air dehydrated samples, respectively. Because vitamin $\mathrm{C}$ is oxygen and heat sensitive, and can be degraded through oxidation even under low oxygen conditions during drying. The rehydration capacity of RF-vacuum dried kiwifruits was significantly higher $(\mathrm{P}<0.05)$ than that in hot air dried samples. The positive vapor pressure pushed from within foods leads to the creation of a porous, loose and fragile texture for the RF dried kiwifruits and therefore results in better water absorption capacity.

Fig. 5 shows the measured moisture distribution in the samples spread over 10 different compartments after the RF-vacuum and hot air drying. For hot air drying, the sample moisture removal was in line with the direction of air flow and resulted in reduced moisture loss as the tray numbers increased from 1 to 10. But the moisture contents of RF-vacuum dehydrated samples were more random and uniform, thereby demonstrating relatively more uniform moisture distribution in kiwifruit slices with RF-vacuum drying. 


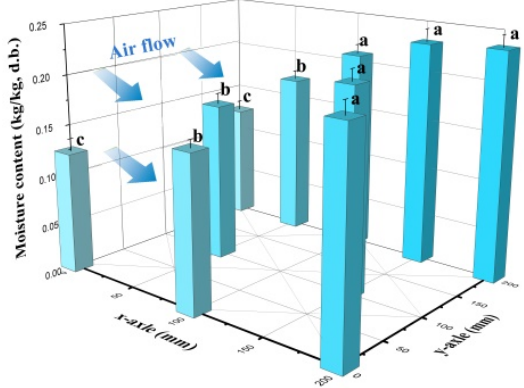

(a)

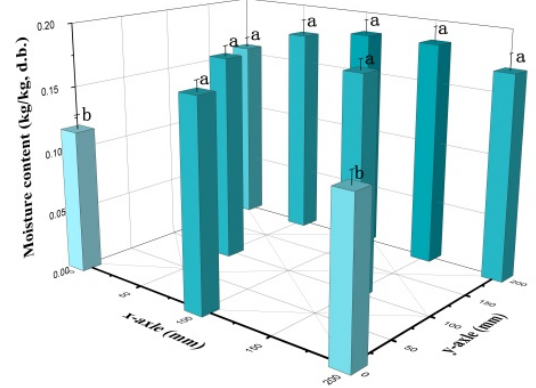

(b)

Fig. 5 Moisture distribution of hot air (a) and RF-vacuum (b) dried kiwifruit samples at 10 compartments in the container with $x$-axis parallel to the hot air flow

\section{Conclusion}

The RF-vacuum drying protocol of RF-vacuum of kiwifruits is electrode gap of $60 \mathrm{~mm}$, vacuum pressure of $0.02 \mathrm{MPa}$ and kiwifruit slice thickness of $8 \mathrm{~mm}$, reducing more than $65 \%$ drying time as compared to AD. Further, the RF-vacuum drying process ensured better quality retention in the dehydrated kiwifruit slices in terms of color, vitamin $\mathrm{C}$ content and rehydration capacity due to fast heating/drying rates and low vacuum pressure. Better uniform moisture distribution was achieved when subjected to the RF-vacuum drying than possible with hot air drying. Overall, RF-vacuum drying technology may provide a more effective and practical dehydration method for kiwifruits with acceptable quality attributes.

\section{References}

[1] FAOSTAT. Food and agriculture organization of the united states 2018 http://www.fao.org/faostat/en/\#data

[2] Mujumdar, A. S. Handbook of industrial drying. Philadelphia, Taylor \& Francis 2007

[3] Ramaswamy, H.; Tang, J. Microwave and radio frequency heating. Food Science and Technology International 2008, 14(5), 423-427.

[4] Zhou, X.; Gao, H.; Mitcham, E. J.; Wang, S. Comparative analyses of three dehydration methods on drying characteristics and oil quality of in-shell walnuts. Drying Technology 2018, 36(4), 477-490.

[5] Huang, Z.; Zhu, H.; Yan, R.; Wang, S. Simulation and prediction of radio frequency heating in dry soybeans. Biosystems Engineering 2015, 129, 34-47.

[6] Maskan, M. Drying, shrinkage and rehydration characteristics of kiwifruits during hot air and microwave drying. Journal of Food Engineering 2001, 48(2), 177-182. 